\title{
Filtering of the interstellar dust flow near the heliopause: the importance of secondary electron emission for the grain charging
}

\author{
Hiroshi Kimura and Ingrid Mann* \\ Max-Planck-Institut für Aeronomie, Max-Planck-Straße 2, D-37191 Katlenburg-Lindau, Germany
}

(Received December 24, 1998; Revised August 24, 1999; Accepted September 17, 1999)

\begin{abstract}
The deflection of interstellar dust grains in the magnetic field near the heliopause depends on their surface electric charge. We study the electric charging of the grains with emphasis on the secondary electron emission because of its importance in the hot plasma environment near the heliopause. We correct previous models of the secondary electron emission that overestimate the electric charge of dust near the heliopause. Our model calculations of the grain charge, when combined with results from in situ measurements of interstellar dust in the heliosphere, place an upper limit on the magnetic field strength. We find that the detection of interstellar dust with mass of $10^{-18} \mathrm{~kg}$ indicates the component of the magnetic field perpendicular to the interstellar dust flow to be less than $0.4 \mathrm{nT}$.
\end{abstract}

\section{Introduction}

The interaction between the very local interstellar medium (VLISM) and the solar wind forms a cavity in the interstellar medium (ISM), which is referred to as the heliosphere (Axford, 1972; Holzer, 1989; Suess, 1990). The relative motion between the solar system and the VLISM produces a flow of interstellar particles towards the heliosphere. While interstellar neutral gas particles enter the heliosphere, some components of the ionized gas are deflected at the heliopause, which is the interface between the VLISM and the heliosphere. Furthermore, interstellar dust grains streaming into the heliosphere along the interstellar downstream direction have been detected (Grün et al., 1994, 1997; Svedhem et al., 1996).

It is worth noting that interstellar dust grains are electrically charged by photoelectron emission, sticking and recombination of plasma particles, secondary electron emission, thermionic emission, and field emission (see Draine (1989) for a review). The relative importance of each charging process depends on the radiation and plasma environment as well as on the size and material composition of the grains. Interstellar dust grains enter a zone of increased plasma temperature between the heliopause and the termination shock, which characterizes the transition of the solar wind flow from supersonic to subsonic (cf. Baranov and Malama, 1993; Pauls and Zank, 1996, 1997; Zank et al., 1996). The high plasma temperature in this region (heliosheath) raises the surface charge of grains as a result of the intensive secondary-electron emission induced by energetic electron bombardment (Kimura and Mann, 1998a). We therefore expect that smaller grains are filtered off in the magnetic field near the heliopause be-

*Also at California Institute of Technology 252-21 Pasadena, California, 91125, U.S.A.

Copy right $(\mathrm{C}$ The Society of Geomagnetism and Earth, Planetary and Space Sciences (SGEPSS); The Seismological Society of Japan; The Volcanological Society of Japan; The Geodetic Society of Japan; The Japanese Society for Planetary Sciences. cause of their high charge-to-mass ratios.

For better estimates of the electric charge on the grains in the high-temperature plasma, this paper presents an improved model of the secondary electron emission from dust grains. The modeling of the secondary electron emission is divided into three parts; the physical process of the secondary electron emission is described along with the energy loss of primary particles in matter expected from experiments of the penetration of electrons and ions into solid materials; the secondary electron emission from a semi-infinite slab is utilized to investigate the validity of the present model by comparison with experiments of the secondary electron emission from a solid bulk; and the secondary electron emission from a sphere is given for the application to the electric charging of interstellar dust near the heliopause. Numerical results are shown for the secondary electron emission from semiinfinite slabs and spherical grains as well as for the electric charge on interstellar dust near the heliopause. We will apply our model calculations of the grain charge to discussions of the orbital deflection of interstellar dust in the magnetic field near the heliopause.

\section{Model Description of Secondary Electron Emis- sion}

\subsection{Elementary theory}

We treat the secondary electron emission induced by electrons in parallel to that induced by ions because of the similarity in their basic physical processes (see Schou, 1988). Our modeling of the secondary electron emission applies an elementary theory, which involves assumptions for the energy loss of the primaries and the generation and transport of the secondaries (Bruining, 1954).

First suppose that primary electrons and ions penetrate into a solid along a straight path and that the number of secondary electrons is proportional to the energy loss of the primaries. The number of secondary electrons generated at a distance $x$ from the entry surface per elementary path length $d x$ is 
expressed as

$$
\frac{1}{\epsilon}\left(-\frac{d E}{d x}\right) d x
$$

where $\epsilon$ and $d E / d x$ denote the energy deposited by the primary into the solid target to produce one secondary electron and the energy loss of the primary per unit path length, respectively.

Secondly we assume that secondary electrons are generated isotropically and that the secondary electrons are subject to exponential absorption with distance in the matter (Jonker, 1952). The probability for secondary electrons to reach the surface per element of angle, $d \varphi$, in the escape direction at an angle $\varphi$ from the direction to the nearest surface is given by

$$
\frac{1}{2} \exp \left(-\frac{l}{\lambda}\right) \sin \varphi d \varphi
$$

where $l$ is the distance to reach the surface of the target from the point where secondary electrons are generated and $\lambda$ is the mean free path of the secondaries in the target.

The key parameter to estimate the secondary electron emission is the secondary electron yield, which is defined by the number of produced secondary electrons per incident primary particle. The secondary electron yield is numerically derived from integrations of Eqs. (1)-(2) over the possible distance $x$ and angle $\varphi$ depending on the geometry of the target as well as the incident direction of primary particles.

\subsection{Energy loss of primaries}

The elementary theory originally relies on a description of the energy loss of primaries given by Whiddington (1912). However, Whiddington's law was confirmed only for the electron energy range above $10 \mathrm{keV}$, but not for low energy ( $\leq 10 \mathrm{keV}$ ) electrons (Young, 1956; Fitting, 1974). Lye and Dekker (1957) have suggested that the energy loss of primary particles is described by the general form,

$$
\frac{d E}{d x}=-A E^{1-n}
$$

where Whiddington's law corresponds to the case of $n=2$. The parameters $A$ and $n$, which depend on the projectile and target, may be determined by measurements of the projected range $R$ as a function of the incident energy $E_{0}$ given by

$$
R\left(E_{0}\right)=(A n)^{-1} E_{0}{ }^{n} .
$$

The definition of the projected range follows as:

$$
R\left(E_{0}\right) \equiv \int_{0}^{E_{0}}\left(-\frac{d E}{d x}\right)^{-1} d E
$$

The projected range $R$ indicates the penetration of a primary particle into matter along the incident direction.

The range-energy relation of electrons in matter for their energy range $0.1 \mathrm{keV} \lesssim E_{0} \lesssim 1 \mathrm{MeV}$ can be approximated by (cf. Fitting, 1974)

$$
R\left(E_{0}\right)=50 \mathrm{~nm}\left(\frac{\rho}{10^{3} \mathrm{~kg} \mathrm{~m}^{-3}}\right)^{-1}\left(\frac{E_{0}}{\mathrm{keV}}\right)^{1.5},
$$

where $\rho$ is the bulk density of the target. Note that only the bulk density is assumed to influence the range-energy relation because the quantity $\rho R$ for different materials falls on a single slope as a function of $E_{0}$.

The projected range of positive ions in the energy range $1 \mathrm{keV} \lesssim E_{0} / \mu_{M_{A}} \lesssim 10 \mathrm{MeV}$ may be given by (cf. Andersen and Ziegler, 1977; Ziegler, 1977, 1980; Santry and Werner, 1986)

$$
\frac{R\left(E_{0}\right)}{\mu_{M_{A}}}=10 \mathrm{~nm}\left(\frac{\rho}{10^{3} \mathrm{~kg} \mathrm{~m}^{-3}}\right)^{-1}\left(\frac{E_{0} / \mu_{M_{A}}}{\mathrm{keV}}\right)^{1.35},
$$

where $\mu_{M_{A}}$ is the mass of the primary ions expressed in atomic mass units. The cases of protons and $\alpha$-particles are therefore taken to be $\mu_{M_{A}}=1.007275$ and $\mu_{M_{A}}=4.00140$, respectively. Draine and Salpeter (1979) assume the projected range for $\alpha$-particles to be $60 \%$ of the proton projected range at the same energy $E_{0}$. This is consistent with Eq. (7) which gives the projected range for $\alpha$-particles to be approximately $62 \%$ of the projected range for protons.

\section{Secondary Electron Emission From a Semi- Infinite Slab}

\subsection{Mathematical formulation}

The secondary electron yield $\delta_{\infty}^{\Theta}\left(E_{0}\right)$ for oblique incidence at an angle $\Theta$ from the normal to the surface is given by (cf. Jonker, 1952):

$$
\begin{aligned}
\delta_{\infty}^{\Theta}\left(E_{0}\right)= & \int_{0}^{x_{\mathrm{m}}} \frac{1}{\epsilon}\left(-\frac{d E}{d x}\right) d x \\
& \times \int_{0}^{\varphi_{\mathrm{c}}} \frac{1}{2} \exp \left(-\frac{l}{\lambda}\right) \sin \varphi d \varphi
\end{aligned}
$$

where $x_{\mathrm{m}}$ is the maximum depth of the primary particles having incident energy $E_{0}$ and $\varphi_{\mathrm{c}}$ is the critical angle for escape. The maximum depth for a semi-infinite slab may be given by $x_{\mathrm{m}}=R$, as long as the slab is considered to be thick compared with the projected range $R$.

In the case of secondary electrons generated at a distance $x$ from the entry of the primary electron into a semi-infinite slab, the distance $l$ is given by

$$
l=x \frac{\cos \Theta}{\cos \varphi} .
$$

The critical angle $\varphi_{\mathrm{c}}$ for a semi-infinite slab may be given by

$$
\cos \varphi_{\mathrm{c}} \equiv\left(\frac{W}{\epsilon}\right)^{\frac{1}{2}}
$$

where $W$ is the potential barrier at the surface. This condition implies that secondary electrons escape if a component of kinetic energy normal to the surface overcomes the potential barrier (Hachenberg and Brauer, 1959). Nevertheless, hereafter we neglect the surface barrier because it is in general so small that the secondary electrons are scarcely influenced (ICRU, 1996).

\subsection{Universal yield curve}

Measured secondary-electron yields for different types of metal slab surfaces as a function of the primary electron energy fall on a single slope, called a universal curve, when the yield is normalized to the maximum yield and the primary electron energy to the energy where the yield is maximized 
(Baroody, 1950). Measurements of the secondary electron emission from polymers induced by electrons are also known to show a universal yield curve (Burke, 1980).

We shall examine how the universal curve is expressed within the framework of this study. If Eqs. (3) and (9) are substituted into Eq. (8), we obtain

$$
\delta_{\infty}^{\Theta}\left(E_{0}\right)=\frac{1}{\epsilon}\left(\frac{A n \lambda}{\cos \Theta}\right)^{\frac{1}{n}} G_{n}\left(\left[\frac{R \cos \Theta}{\lambda}\right]^{\frac{1}{n}}\right),
$$

where

$$
G_{n}(r) \equiv \frac{1}{2} \int_{0}^{1} z^{\frac{1}{n}} \exp \left(-\frac{r^{n}}{z}\right) d z \int_{0}^{r / z^{\frac{1}{n}}} \exp \left(y^{n}\right) d y .
$$

The function $G_{n}(r)$ of variable $r$ has a single maximum $G_{n}\left(r_{\mathrm{m}}\right)$ at the variable $r_{\mathrm{m}}$. Figure 1 shows the variable $r_{\mathrm{m}}$ and the maximum $G_{n}\left(r_{\mathrm{m}}\right)$ against the power index $n$ of the rangeenergy relation given in Eq. (4). Numerical estimates for Eq. (12) performed by the Romberg integration bring about $G_{n}\left(r_{\mathrm{m}}\right)=0.167$ at $r_{\mathrm{m}}=0.898$ for electrons $(n=1.5)$ and $G_{n}\left(r_{\mathrm{m}}\right)=0.174$ at $r_{\mathrm{m}}=1.09$ for positive ions $(n=1.35)$.

The mean free path $\lambda$ and the dissipation energy $\epsilon$ are given by

$$
\begin{aligned}
\lambda & =(A n)^{-1}\left(E_{\mathrm{m}} / r_{\mathrm{m}}\right)^{n}, \\
\epsilon & =\left(E_{\mathrm{m}} / \delta_{\mathrm{m}}\right)\left[G_{n}\left(r_{\mathrm{m}}\right) / r_{\mathrm{m}}\right],
\end{aligned}
$$

where $\delta_{\mathrm{m}}$ is the maximum yield peaking at primary particle energy $E_{\mathrm{m}}$ for normal incidence (i.e., $\Theta=0$ ).

Equation (11) can be turned into the universal yield curve:

$$
\delta_{\infty}^{\Theta}\left(E_{0}\right) / \delta_{\mathrm{m}}^{\Theta}=G_{n}\left(\frac{E_{0}}{E_{\mathrm{m}}^{\Theta}} r_{\mathrm{m}}\right) / G_{n}\left(r_{\mathrm{m}}\right)
$$

where $\delta_{\mathrm{m}}^{\Theta}$ is the maximum yield at energy $E_{\mathrm{m}}^{\Theta}$ for oblique incidence with an angle $\Theta$. The yield $\delta_{\mathrm{m}}^{\Theta}$ and the energy

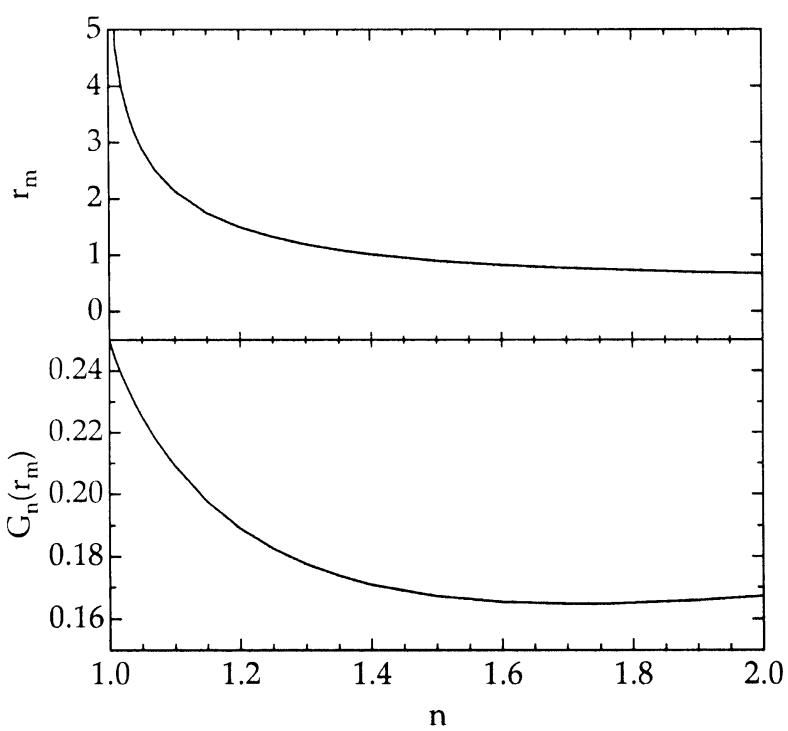

Fig. 1. The variable $r_{\mathrm{m}}$ at which $G_{n}(r)$ given in Eq. (12) is maximized and its maximum value $G_{n}\left(r_{\mathrm{m}}\right)$ are shown as a function of the power index $n$.
$E_{\mathrm{m}}^{\Theta}$ are given by $\delta_{\mathrm{m}}^{\Theta}=\delta_{\mathrm{m}} \cos ^{-\frac{1}{n}} \Theta$ and $E_{\mathrm{m}}^{\Theta}=E_{\mathrm{m}} \cos ^{-\frac{1}{n}} \Theta$ respectively.

\subsection{Other models for a yield curve}

When $n=2$ (i.e., Whiddington's law) is assumed in Eq. (15), the present model for the universal yield curve is identical to the theory by Jonker (1952). Sternglass (1957) constructed another model for the electron-induced yield curve of metal slab surfaces; $\delta_{\infty}\left(E_{0}\right) / \delta_{\mathrm{m}}=e^{2}\left(E_{0} / E_{\mathrm{m}}\right)$. $\exp \left\{-2\left(E_{0} / E_{\mathrm{m}}\right)^{1 / 2}\right\}$. Draine and Salpeter (1979) have proposed an empirical fitting formula for the measured yield curve: $\delta_{\infty}\left(E_{0}\right) / \delta_{\mathrm{m}}=4\left(E_{0} / E_{\mathrm{m}}\right) /\left(1+E_{0} / E_{\mathrm{m}}\right)^{2}$. These two models are often used to estimate the secondary electron emission from cosmic dust in a high-temperature plasma (Mukai, 1981; Meyer-Vernet, 1982; Havnes et al., 1992; Horányi et al., 1995). Jurac et al. (1995) have shown that experiments with a water ice slab are in good agreement with the empirical formula of Draine and Salpeter (1979), but not with the theory of Sternglass (1957).

For the proton-induced yield curve at normal incidence, Katz et al. (1977) show a simple formula based on a constant energy loss as $\delta_{\infty}\left(E_{0}\right) / \delta_{\mathrm{m}}=2\left(E_{0} / E_{\mathrm{m}}\right)^{1 / 2} /\left(1+E_{0} / E_{\mathrm{m}}\right)$. Draine and Salpeter (1979) suggest another expression of the proton-induced yield curve as $\delta_{\infty}\left(E_{0}\right)=$ $0.1\left(1+E_{0} / 500 \mathrm{eV}\right) /\left(1+E_{0} / 35 \mathrm{keV}\right)^{2}$, which implies that the yield is maximized at the same energy $E_{\mathrm{m}}=34 \mathrm{keV}$ to be always $\delta_{\mathrm{m}}=1.775$, independent of the material composition of the target.

\subsection{Electron-induced yield curve}

Figure 2 shows the theoretical universal curve of the secondary electron emission from a semi-infinite slab for normal incidence of electrons in comparison with experimental data for silicate and carbon (Salow, 1940; Yong et al., 1998; Bruining, 1954). While the theory of Jonker (1952) is lower than experimental results over the entire energy range, the theory of Sternglass (1957) fails to fit the ex-

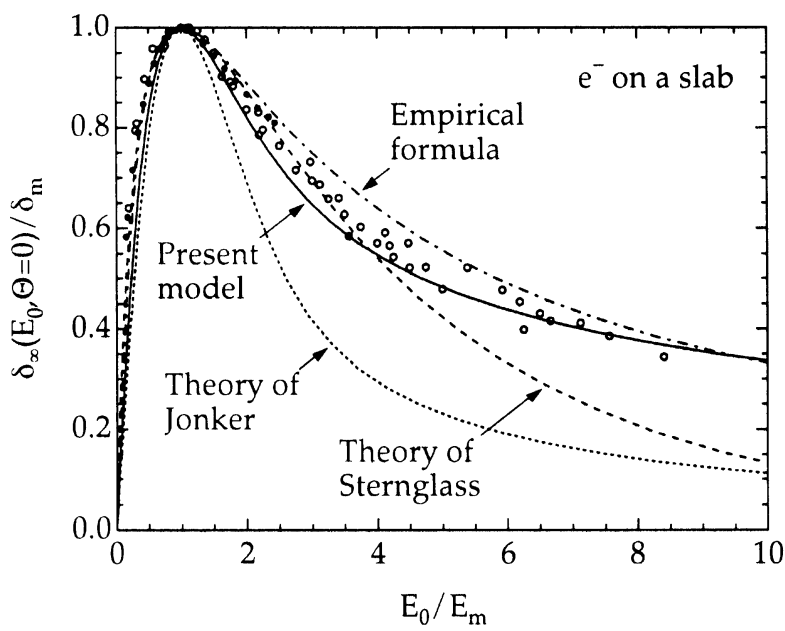

Fig. 2. The secondary electron yield from a semi-infinite slab due to normal incidence of electrons are compared between models and experiments. Solid, dotted, dashed, and dash-dotted lines indicate present model, theory of Jonker (1952), theory of Sternglass (1957), and empirical formula of Draine and Salpeter (1979), respectively. Open circles denote experimental data of silicate by Salow (1940) and Yong et al. (1998). Closed circles denote experimental data of carbon given in Bruining (1954). 
perimental data for $E_{0} / E_{\mathrm{m}}>4$. The empirical formula of Draine and Salpeter (1979) shows an overall agreement, but slightly overestimates experimental data for the energy range $E_{0} / E_{\mathrm{m}}>1$. The agreement of the present model curve with experimental results is fairly good over the whole energy range of incident electrons, although the present model tends to underestimate the measured yield values.

\subsection{Proton-induced yield curve}

Figure 3 shows a comparison between models and measurements of the secondary electron emission from a slab under proton bombardment with $1 \leq E_{0} / E_{\mathrm{m}} \leq 10$. The slope of the yield curve derived from experiments is steeper than that predicted by the theory of Katz et al. (1977). On the contrary, experimental data show a gentler slope compared with the yield curve given by the empirical formula of Draine and Salpeter (1979) for $1 \leq E_{0} / E_{\mathrm{m}} \leq 4$. Clearly, the experimental data are best fitted by the present model than by either the theory of Katz et al. (1977) or the empirical formula of Draine and Salpeter (1979).

The angular variation in the secondary electron yield can also be compared between the models and experimental data. The present model predicts the angular dependence of the yield to be $\cos ^{-0.74} \Theta$ for proton impacts. This is consistent with the experimental yields for a silicate film indicating that the power of $\cos \Theta$ ranges from -0.85 to -0.70 (Jacobsson, 1993; Jacobsson and Holmén, 1993).

\subsection{Validity of the present model}

In contrast to our assumptions, the power index $n$ and fitting parameter $A$ of the range-energy relation is, in general, not constant over a wide energy range of the primary particles (see Kanaya et al., 1978). Furthermore, measured yield data involve the primary electrons reflected at the sur-

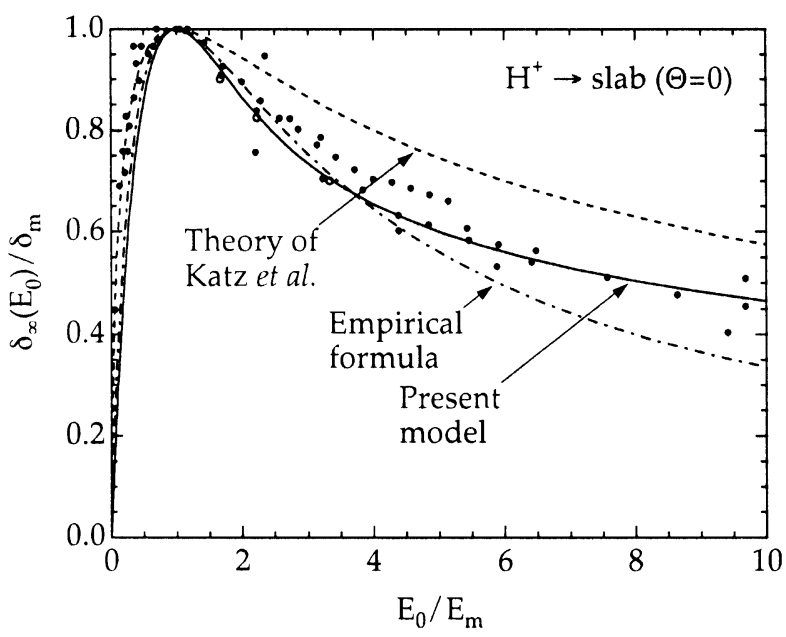

Fig. 3. The same as Fig. 2, but for proton impact. Solid, dotted, dash-dotted lines indicate present model, theory of Katz et al. (1977), and empirical formula of Draine and Salpeter (1979), respectively. Open circles denote experimental data of silicate by Jacobsson (1993) and Jacobsson and Holmén (1994) with assumptions of $\delta_{\mathrm{m}}=4.0$ and $E_{\mathrm{m}}=90 \mathrm{keV}$ from the similarity of the slopes between the secondary electron yield and the stopping power (ICRU, 1993). Closed circles denote experimental data of carbon by Meckbach et al. (1975) with $\delta_{\mathrm{m}}=4.0$ at $E_{\mathrm{m}}=20 \mathrm{keV}$, Hasselkamp et al. (1990) with $\delta_{\mathrm{m}}=1.7$ at $E_{\mathrm{m}}=85 \mathrm{keV}$, Gelfort et al. (1997) with $\delta_{\mathrm{m}}=3.24$ at $E_{\mathrm{m}}=70 \mathrm{keV}$, and Ritzau and Baragiola (1998) with $\delta_{\mathrm{m}}=2.9$ at $E_{\mathrm{m}}=85 \mathrm{keV}$. face of a slab and those scattered backward in the slab (see Hachenberg and Brauer, 1959). The model yield curve could be better described by taking into account these two effects. The present model, however, yields a good fit to the data within the uncertainties of the experimental results for both electrons and protons.

\section{Secondary Electron Emission From a Spherical Grain}

\subsection{Mathematical formulation}

Draine and Salpeter (1979) have suggested that the geometry of small particles compared to infinite slabs leads to a higher emission of secondary electrons, which is referred to as the small particle effect. The Jonker's theory (the elementary theory with the Whiddington's law) has been extended to treat the small particle effect of electron impact on small spherical grains (Chow et al., 1993, 1994; Chow, 1997). We reconsider the formulation of the small particle effect using Eqs. (6) and (7), which give a better description of the experimental data, rather than the Whiddington's law. The elementary theory has also been used to investigate the influence of the porosity on the electron-induced secondary-electron emission (Millet and Lafon, 1995). While the porosity effect on the secondary electron emission from dust grains can be revised in the framework of the present model, this is beyond the scope of this paper.

When a spherical grain with radius $a$ is immersed in an isotropic flux of electrons or ions, the secondary electron yield $\delta_{a}\left(E_{0}\right)$ from the grain is given by (cf. Chow, 1997)

$$
\begin{aligned}
\delta_{a}\left(E_{0}\right)= & \int_{0}^{\pi / 2} \sin \Theta \cos \Theta d \Theta \\
& \times \int_{0}^{x_{\mathrm{m}}} \frac{1}{\epsilon}\left(-\frac{d E}{d x}\right) d x \int_{0}^{\pi} \exp \left(-\frac{l}{\lambda}\right) \sin \varphi d \varphi,
\end{aligned}
$$

where $d E / d x, \lambda$, and $\epsilon$ are determined by Eqs. (3), (13), and (14) respectively on the assumption that these quantities are independent of the shape and structure of the target. We here notice that the secondary electron yield for a spherical grain under a mono-directional flux of electrons or ions is identical to the case of an isotropic flux because of the symmetrical shape of a sphere. The distance $l$ to the grain's surface is given by

$$
l=a\left[\sqrt{1-(u \sin \varphi)^{2}}-u \cos \varphi\right]
$$

where

$$
u=\sqrt{1+(x / a)^{2}-2(x / a) \cos \Theta} .
$$

The maximum depth $x_{\mathrm{m}}$ of primary is limited by either the projected range of the primaries or the size of the dust grain, namely,

$$
x_{\mathrm{m}}=\min (R, 2 a \cos \Theta),
$$

where $\Theta$ is an angle between the incident direction of the primary and the normal to the surface of the grain.

\subsection{Material composition of interstellar dust}

The properties of dust grains in the VLISM have been suggested to be similar to those in the diffuse ISM (Reach and 


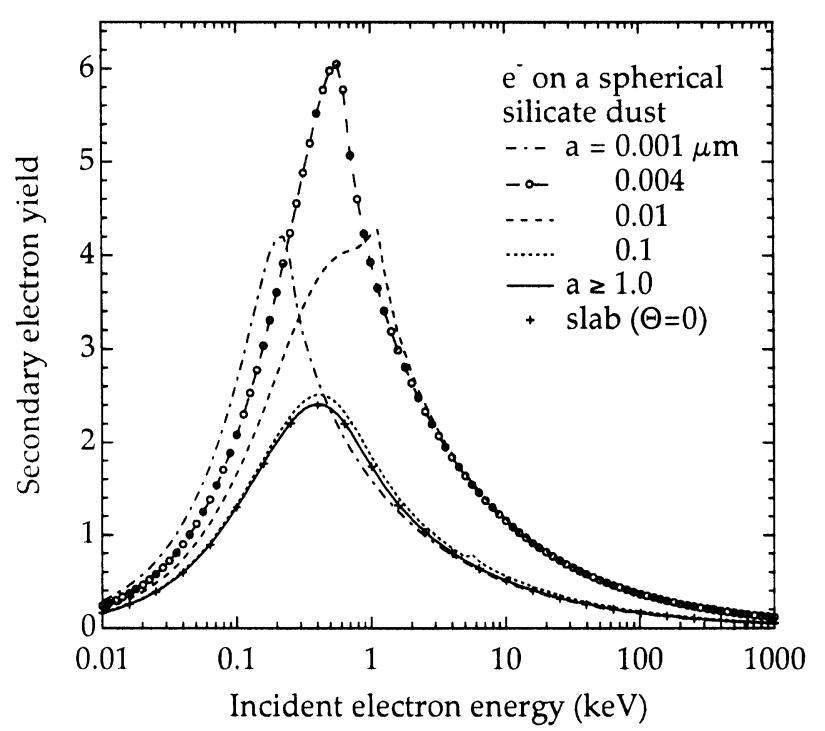

Fig. 4. The secondary electron yield from a spherical silicate grain by electron impact is given as a function of incident electron energy ranging from 0.01 to $1000 \mathrm{keV}$. The yield curves are shown for grain radius $a=0.001,0.004,0.01$, and $0.1 \mu \mathrm{m}$, separately, and those for $a \geq 1.0 \mu \mathrm{m}$ converge on the yield curve for the solid slab surface.

Boulanger, 1998; Kimura et al., 1999). Although there is no definite model of interstellar dust up to present, there is a consensus of the existence of silicate and carbon materials among different component of dust in the diffuse ISM (Greenberg, 1978; Draine and Lee, 1984; Jones et al., 1987; Mathis and Whiffen, 1989; see, Dorschner (1996) for a review). Although Frisch et al. (1999) suggest a model of core-mantle grains with olivine and oxide in the core and pyroxene in the mantle within a few parsec from the Sun, we assume silicate and carbon to simulate the material properties of dust in the VLISM.

The bulk densities $\rho$ for silicate and carbon grains are given by $\rho=3.3 \times 10^{3}$ and $\rho=2.25 \times 10^{3} \mathrm{~kg} \mathrm{~m}^{-3}$ respectively. The yield parameters $\delta_{\mathrm{m}}$ and $E_{\mathrm{m}}$ for electron bombardment on a slab have been experimentally determined to be $\delta_{\mathrm{m}}=2.4$ at $E_{\mathrm{m}}=400 \mathrm{eV}$ for silicate and $\delta_{\mathrm{m}}=1$ at $E_{\mathrm{m}}=250 \mathrm{eV}$ for carbon (Kollath, 1956; Hachenberg and Brauer, 1959). Substituting the yield parameters into Eqs. (13) and (14), we obtain the mean free path $\lambda$ and the dissipation energy $\epsilon$ for electron bombardment to be $\lambda=4.5 \mathrm{~nm}$ and $\epsilon=31 \mathrm{eV}$ for silicate grains and $\lambda=3.3 \mathrm{~nm}$ and $\epsilon=46.5 \mathrm{eV}$ for carbon grains. Our estimate for the mean free path $\lambda$ of the secondary electrons in carbon is in a good agreement with the experimental value (cf. Voreades, 1976).

\subsection{Theoretical yield curve}

Figures 4 and 5 show the calculated yield curve of the secondary electron emission induced by electron impact onto spherical silicate and carbon grains in comparison to semiinfinite slabs due to normal incident electrons. Unfortunately, there is no experimental data for the secondary electron yield of silicate nor carbon grains.

The values of the secondary electron yield from large spherical grains with $a>1 \mu \mathrm{m}$ show no further variation with the size of grains and are identical to those from a semi-infinite slab due to normal incidence. This is consis-

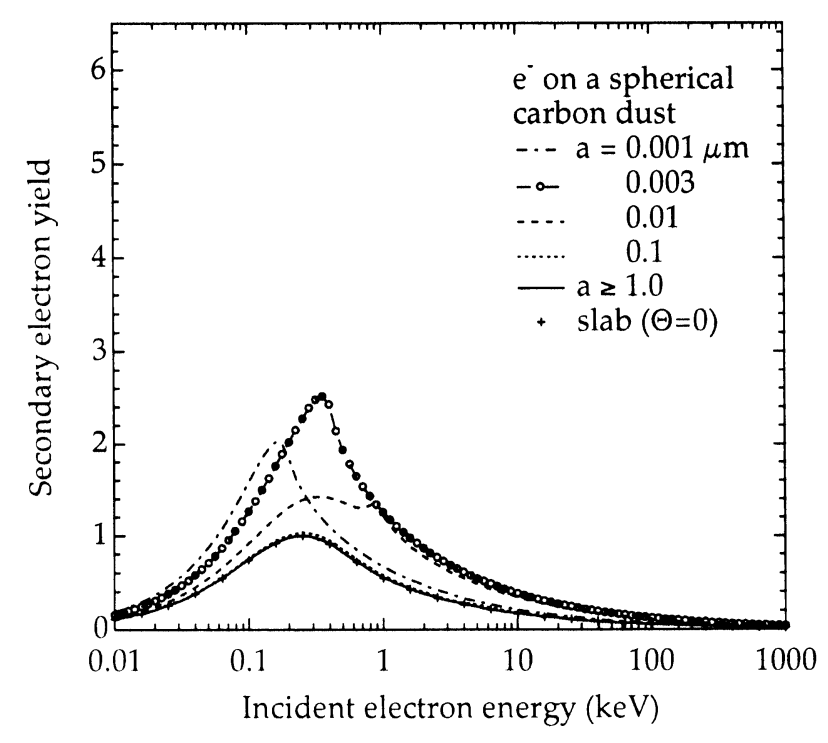

Fig. 5. The same as Fig. 4 but for a spherical carbon grain with $a=0.001$, $0.003,0.01,0.1 \mu \mathrm{m}$, and $\geq 1.0 \mu \mathrm{m}$.

tent with measurements of the secondary electron emission from polystyrene latex spheres of submicron size, the secondary electron yield of which has been shown to agree with the value measured for polystyrene foils (Hall and Beeman, 1976; Ziemann et al., 1995). In contrast, the empirical formula of Draine and Salpeter (1979) gives a value of two times higher in the large-size limit.

Grains with $a=0.1 \mu \mathrm{m}$ show a yield curve similar to, but slightly higher than that of larger grains with $a=1 \mu \mathrm{m}$, over all energy ranges of primary electrons. Furthermore, an additional small peak appears in the yield curve at $E_{0}=$ $5.6 \mathrm{keV}$ for silicate and $E_{0}=4.3 \mathrm{keV}$ for carbon grains with $a=0.1 \mu \mathrm{m}$. The projected ranges at these energies are found for both silicate and carbon grains to be $R\left(E_{0}\right)=0.2 \mu \mathrm{m}$, which corresponds to the diameter $2 a$ of the grains. A similar second peak at $E_{0}>E_{\mathrm{m}}$ has also been found in the yield curve of the secondary electron emission from surfaces of thin carbon foils (see Caron et al., 1998). While the energy of the additional peak decreases with decreasing size of grains, the yield curve for grains with $a \geq 0.01 \mu \mathrm{m}$ shows that the incident electron energy for the additional peak is higher than $E_{\mathrm{m}}$. The second peak for $a=0.01 \mu \mathrm{m}$ occurs at $E_{0}=$ $1.1 \mathrm{keV}$ with $\delta=4.3$ for silicate and $E_{0}=0.89 \mathrm{keV}$ with $\delta=1.4$ for carbon. The projected ranges for these energies are given by $R\left(E_{0}\right) \approx 0.02 \mu \mathrm{m}$ for both silicate and carbon grains. On the other hand, the projected ranges $R$ for $E_{\mathrm{m}}$ amount to $0.0038 \mu \mathrm{m}$ for silicate grains and $0.0028 \mu \mathrm{m}$ for carbon grains. As far as the grain diameter $2 a$ is larger than the range $R\left(E_{\mathrm{m}}\right)$, the second peak occurs at the incident electron energy where the range $R$ reaches the diameter $2 a$ of the grain.

The small particle effect becomes significant for grains with $a<0.1 \mu \mathrm{m}$ and causes an enhancement in the yield. Very small grains with $a=0.001 \mu \mathrm{m}$ have a single peak in the yield curve, which is $\delta=4.2$ at $E_{0}=0.21 \mathrm{keV}$ for silicate and $\delta=2.0$ at $E_{0}=0.17 \mathrm{keV}$ for carbon. The peak value of the yield attains the highest at radii $a \approx 0.004 \mu \mathrm{m}$ for 
silicate grains and at radii $a \approx 0.003 \mu \mathrm{m}$ for carbon grains. One may notice that the yield curve of a semi-infinite slab is maximized at the projected range $R\left(E_{\mathrm{m}}\right)=0.0038 \mu \mathrm{m}$ for silicate and $R\left(E_{\mathrm{m}}\right)=0.0028 \mu \mathrm{m}$ for carbon. Therefore, the highest value for the secondary electron yield is obtained when the radius corresponds to the projected range of the energy at which the yield of a semi-infinite slab is maximized, namely, when $a \approx R\left(E_{\mathrm{m}}\right)$ is satisfied. The highest yields of spherical silicate and carbon grains are found to be $\delta \approx 6$ and $\delta \approx 2.5$ respectively and are a factor of 2.5 higher than for large $(a \geq 1 \mu \mathrm{m})$ grains.

\section{Application to Grain Charging}

\subsection{Charging processes for interstellar dust}

We apply the present model for the secondary electron emission from a sphere to estimate the electric charge of dust streaming from the VLISM to the heliosphere. As possible charging processes, photoelectron emission, sticking of plasma particles, and secondary electron emission are considered, while thermionic emission and field emission are negligible near the heliopause (cf. Kimura and Mann, 1999). Penetration of energetic plasma and the recombination of ions with electrons on the dust surface are taken into account for the estimate of the plasma sticking current. The method of computing each current is described in Kimura and Mann (1998a), while Eq. (16) is applied to estimate the secondary electron yield, for comparison to the previously used formula that is empirically derived by Draine and Salpeter (1979). We find the dust charge for which the sum of the currents equals to zero as the equilibrium condition.

Note that not only the secondary electron emission but also the photoelectron emission weakly reflects dust materials through the dielectric function and photoelectric yield of grains (Draine 1978; Draine and Salpeter, 1979; Kimura and Mann, 1998a). The dielectric functions of silicate and carbon materials are taken from Laor and Draine (1993) and the mean free path $\lambda$ of the secondary electrons is used to compute the photoelectric yield (Draine and Salpeter, 1979; Kimura and Mann, 1998a). It is worth mentioning that except for the electron sticking current, the photoelectron current is known to contribute significantly to the electric charging in the inner solar system, but the secondary electron current is predominant near the heliopause.

\subsection{Radiation and plasma environments}

The photon flux used for calculations of the photoelectron current is given by Mezger et al. (1982) for interstellar background stars and by Heroux et al. (1974), Higgins (1976), and Vernazza et al. (1976) for the Sun. The major constituent of the plasma near the heliopause is protons with high temperature resulting from their compression at the termination shock. We adopt $n_{p}=1.87 \times 10^{3} \mathrm{~m}^{-3}$, $T_{p}=2 \times 10^{6} \mathrm{~K}$, and $v_{p}=0 \mathrm{~km} \mathrm{~s}^{-1}$ where $n_{p}, T_{p}$, and $v_{p}$ denote the number density, temperature, and heliocentric radial bulk speed of protons respectively (cf. Pauls and Zank, 1996). Although $\alpha$-particles are of minor importance for the electric charging of dust, we assume the number density $n_{\alpha}$ of the $\alpha$-particles $n_{\alpha}=0.05 n_{p}$ and its temperature and bulk speed to be the same as those of protons. We do not take into account the heavier elements because their contributions are even smaller. Hence the plasma condition $n_{e}=n_{p}+2 n_{\alpha}$

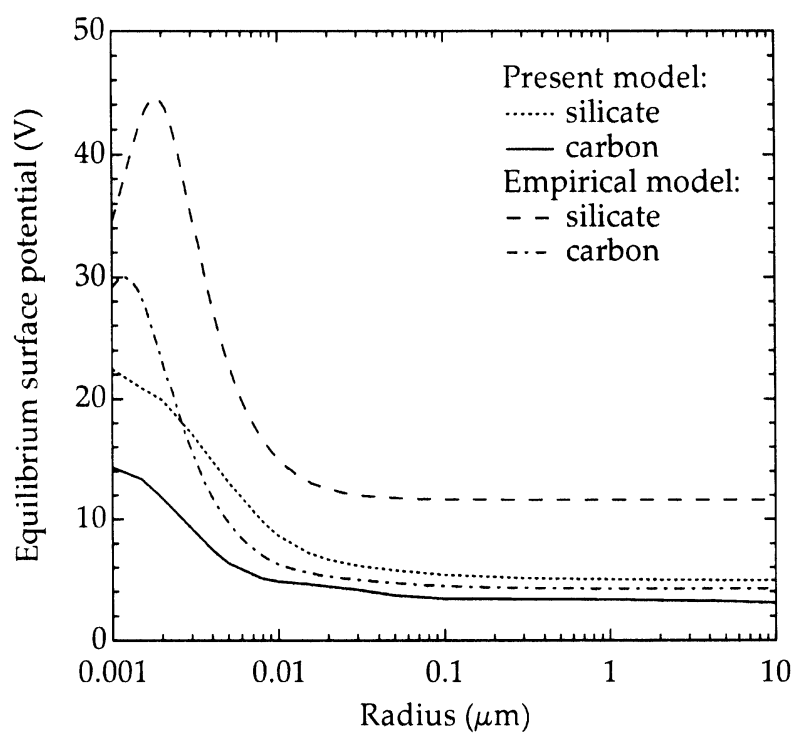

Fig. 6. The equilibrium surface potentials of interstellar dust near the heliopause are enhanced for nanometer-sized grains because of the small particle effect. The dotted and solid curves are numerical potentials of silicate and carbon grains that use Eq. (16) with the range-energy relation given in Eq. (6). The dashed and dash-dotted curves indicate the potentials for silicate and carbon grains calculated with the empirical formula suggested by Draine and Salpeter (1979) for the secondary electron emission from small spherical particles due to electron bombardment.

determines the electron number density $n_{e}$, while the temperature and bulk speed of the electrons are assumed to be identical to $T_{p}$ and $v_{p}$ respectively.

\subsection{Electric charge on interstellar dust}

The electric charge $Q$ on the surface of a spherical grain can be expressed in terms of its surface potential $U$ using the relation $Q=4 \pi \epsilon_{0} a U$, where $\epsilon_{0}$ is the permittivity in vacuum. Figure 6 shows the calculated surface potentials of interstellar dust grains based on the discussed assumptions in comparison to previous calculations. The present model for the secondary electron emission results in lower surface potentials over the whole size range. While silicate grains attain potentials higher than carbon grains, the difference of the potentials in the present model is smaller (e.g., $\sim 2 \mathrm{~V}$ for $a>0.1 \mu \mathrm{m}$ ) compared to the previous model (e.g., $\sim 7 \mathrm{~V}$ for $a>0.1 \mu \mathrm{m})$. These results indicate that the effect of the secondary electron emission on the electric charging is less significant than previously estimated.

The difference in the surface potentials of grains between silicate and carbon increases at the small size range where the small particle effect pronounces the contribution of the secondary electron emission to the grain charging. In the previous model, the small particle effect considerably enhances the potentials of small grains for $a<0.01 \mu \mathrm{m}$ accompanied with a peak at the size where the secondary electron yield reaches its maximum. In contrast, the present model indicates a gradual increase in the surface potential for grain sizes $a<0.1 \mu \mathrm{m}$ without showing a maximum at the position of the maximum secondary-electron yield. The absence of the peak in the potential curve suggests that the feature of the secondary electron emission is diluted by the pene- 
tration of the impinging electrons, which becomes effective monotonically with decreasing size of grains.

\section{Discussion}

6.1 Filtering of interstellar dust flow near the heliopause

The depletion mechanism of grains due to the electric charges may be less significant than previously expected, since the present model predicts lower values of grain charges (see Fig. 6). In order to discuss the deflection of interstellar dust by the Lorentz force between the heliopause and the termination shock, we compare the initial momentum of the grains with the incremental momentum change of grains as a result of the interaction between the dust charge and the magnetic field. The condition of $m v_{\infty}>\int Q v B \mathrm{~d} t$ may, therefore, be regarded as an indicator for the undisturbed flow of interstellar dust with mass $m$ and initial heliocentric speed $v_{\infty}$ (cf. Kimura and Mann, 1998b). We assume the initial speed of interstellar dust to be $v_{\infty}=26 \mathrm{~km} \mathrm{~s}^{-1}$, which was derived from the speed distribution of interstellar dust detected in the heliosphere (Grün et al., 1994). The electric charge $Q$ and speed $v$ of the grain relative to the plasma, and the magnetic field strength $B$ in the direction perpendicular to the initial dust velocity are in general described as a function of time $t$ along the trajectory of the interstellar dust. However, the electric charges on grains in the equilibrium state are almost constant in the heliosheath (Kimura and Mann, 1998a,b; Mann and Kimura, 1999). On the other hand, the speed of the grains relative to the plasma increases with time according to the spatial variation in the solar wind speed varying from $0 \mathrm{~km} \mathrm{~s}^{-1}$ at the heliopause to approximately $100 \mathrm{~km} \mathrm{~s}^{-1}$ at the termination shock. As interstellar dust grains travel from the heliopause to the termination shock, the magnetic field strength decreases (cf. Linde et al., 1998). Assuming a constant dust charge and a monotonic increase in the relative speed and decrease in the magnetic field in the time interval $0 \leq t \leq L / v_{\infty}$, one can obtain the inequality $\int Q v B \mathrm{~d} t>Q v_{\mathrm{HP}} B_{\mathrm{HP}} L / v_{\infty}$ where $v_{\mathrm{HP}}$ and $B_{\mathrm{HP}}$ denote the dust speed relative to the plasma and the magnetic field strength at the heliopause and $L$ the distance between the heliopause and the termination shock. Thus the condition for the undisturbed flow of interstellar dust can be transformed into $m v_{\infty} / Q>B_{\mathrm{HP}} L$ because of $v_{\mathrm{HP}}=v_{\infty}$. Note that the estimates of the grain charge $Q$ for the smallest grains of mass $m$ detected in the heliosphere determine the upper limit on $B_{\mathrm{HP}} L$.

Data for in situ dust experiments are available at present from 1989 to 1995 for Galileo between 0.7-5.3 AU from the Sun and from 1990 to 1995 for Ulysses between 1.05.4 AU (Grün et al., 1995b,c; Krüger et al., 1999a,b). The uncertainty of the mass determination may deviate two orders of magnitude from the real value (Göller and Grün, 1989; Grün et al., 1995a). Nevertheless the data analysis indicates the detection of interstellar dust grains with masses as small as $m=1 \times 10^{-18} \mathrm{~kg}$ (Baguhl et al., 1996; Mann and Kimura, 1999). Since their radii can be in the range of $0.039-0.062 \mu \mathrm{m}$ assuming the specific density of $1 \times 10^{3} \leq \rho \leq 4 \times 10^{3} \mathrm{~kg} \mathrm{~m}^{-3}$, their surface potentials of $U>3.5 \mathrm{~V}$ (see Fig. 6) allow us to derive the heliosheath condition $B_{\mathrm{HP}} L<11.4 \mathrm{nT}$ AU. The distance $L$ between the termination shock and the heliopause seems to be greater than $30 \mathrm{AU}$ in the upstream direction with respect to the interstellar wind, based on different models of the interaction between the solar wind and the VLISM (Whang et al., 1995; Baranov and Malama, 1996; Washimi and Tanaka, 1996; Zank and Pauls, 1996; Linde et al., 1998; Ratkiewicz et al., 1998). This places further constraints of $B_{\mathrm{HP}}<0.38 \mathrm{nT}$ on the perpendicular component of the magnetic field at the heliopause as far as the condition $L>30 \mathrm{AU}$ is fulfilled.

The value of $0.38 \mathrm{nT}$ can also be regarded as the upper limit on the perpendicular component of the magnetic field in the VLISM, if the magnetic field strength at the heliopause is stronger than that in the VLISM as derived from many models and this is true for the magnetic field component (see, Nerney et al., 1993; Washimi and Tanaka, 1996; Linde et al., 1998). This is consistent with the value of $0.43 \mathrm{nT}$ that has been estimated as an upper limit on the magnetic field strength in the VLISM (see Gloeckler et al., 1997). Our calculations of the grain charge give us another possibility to estimate the upper limits of the magnetic field in the heliospheric boundary and the VLISM.

\subsection{Deflection of interstellar dust flow at the bow shock}

Depending on the grain charge and the magnetic field, tiny dust grains could already be depleted near the bow shock, which can be expected to form outside the heliopause if the interstellar gas flow is supersonic (cf. Baranov et al., 1976). Frisch et al. (1999) have recently concluded that interstellar dust grains with $a<0.01 \mu \mathrm{m}$ are excluded at the bow shock, based on the condition that the Larmor radius of the grains at the bow shock is smaller than the distance between the bow shock and the heliopause. The Larmor radius $(m v / Q B)$ was derived for graphite grains $\left(\rho=2.26 \times 10^{3} \mathrm{~kg} \mathrm{~m}^{-3}\right)$ from roughly estimated surface charge $Q$ and adopted values of $v=26 \mathrm{~km} \mathrm{~s}^{-1}$ and $B=0.3 \mathrm{nT}$. The value for the magnetic field comes from assumptions of the compression ratio $\sim 2$ at the bow shock and the interstellar magnetic field of $0.15 \mathrm{nT}$. Because $v \sim 13 \mathrm{~km} \mathrm{~s}^{-1}$ for the case of the compression ratio $\sim 2$, the Larmor radius at the bow shock was overestimated. While Frisch et al. (1999) assume implicitly that the interstellar dust flow is undisturbed outside the bow shock (i.e., $v=0 \mathrm{~km} \mathrm{~s}^{-1}$ ), it may be worth investigating the probability for interstellar dust grains with $a<0.01 \mu \mathrm{m}$ to reach the bow shock without a significant deflection. The dispersion speed of grains has been derived from experimental data in the heliosphere to be $5 \mathrm{~km} \mathrm{~s}^{-1}$ and the electric charge $Q$ of grains with $a<0.01 \mu \mathrm{m}$ is almost constant outside the heliopause (Grün et al., 1994; Kimura and Mann, 1998a). If $B=0.15 \mathrm{nT}$, the derived Larmor radius of the graphite grains with $a<0.01 \mu \mathrm{m}$ outside the bow shock is as small as $58 \mathrm{AU}$, which is much smaller than the scale of the local interstellar cloud. Consequently, the assumed values for the estimate of the Larmor radius would lead to the conclusion that interstellar dust grains with $a<0.01 \mu \mathrm{m}$ do not reach the bow shock as well as the heliopause.

We notice that a comparison of the Larmor radius with the distance between the bow shock and heliopause is not a good indicator for the deflection of interstellar dust flow; when the speed $v$ of interstellar dust grains relative to the interstellar wind is small, the resulting small value of the Larmor radius might be misunderstood as the significant deflection of the grains despite of the ineffective Lorentz force. Instead, 
we apply the inequality $m v_{\infty}^{2} / Q v>B L$ to investigate the condition for the undisturbed flow of interstellar dust having the charge $Q$ in the distance $L$ between the bow shock and the heliopause. The surface potential of grains with mass $m=1 \times 10^{-18} \mathrm{~kg}$ fulfills $U>2.7 \mathrm{~V}$ at the bow shock (cf. Mann and Kimura, 1999). If $L=150 \mathrm{AU}$ is assumed for the distance between the bow shock and the heliopause according to Frisch et al. (1999), the detection of interstellar dust with $m=1 \times 10^{-18} \mathrm{~kg}$ in the heliosphere would indicate the condition of $B<0.16 \mathrm{nT}$ for the interstellar magnetic strength at the bow shock. However, the upper limit on the interstellar magnetic field outside the bow shock is turned out from the compression ratio $\sim 2$ at the bow shock to be $0.08 \mathrm{nT}$, which contrasts with the value of $0.15 \mathrm{nT}$ assumed by Frisch et al. (1999). It is worth noting that the strength and direction of the magnetic field in the VLISM are poorly known at present (Holzer, 1989; Suess, 1990; Frisch, 1995). In addition, the bow shock may not exist at all because of the subsonic interstellar flow, which seems to be indicated by a comparison of heliospheric models with the Ly $\alpha$ absorption profile toward $\alpha$ Cen (Gayley et al., 1997). It can also be dissipated by collisions between ions and neutrals in the interstellar magnetic field or it may disappear during the ascending phase of the solar cycle (Mullan and Arge, 1996; Richardson, 1997). In order to answer the question whether the interstellar dust flow is filtered off at the bow shock or not, further studies of the interstellar magnetic field along with the existence of the bow shock are required. In addition, numerical simulations of dust trajectories compared with measurements of the dust velocity and mass distributions in the heliosphere may put constraints on the interstellar magnetic field.

Acknowledgments. We thank Priscilla Frisch and an anonymous referee for thoughtful review reports, Alexander Krivov, James F. McKenzie, Romana Ratkiewicz, and Paul J. Ziemann for fruitful communications, and Tadashi Mukai for useful discussions and financial support for one of us (H. K.) at Kobe University. This work was supported by BMBF.

\section{References}

Andersen, H. H. and J. F. Ziegler, Hydrogen: Stopping Powers and Ranges in All Elements, 317 pp., Pergamon Press, New York, 1977.

Axford, I. W., The interaction of the solar wind with the interstellar medium, in Solar Wind, edited by C. P. Sonett, P. J. Coleman, Jr., and J. M. Wilcox, pp. 609-660, NASA SP-308, Washington, D. C., 1972.

Baguhl, M., E. Grün, and M. Landgraf, In situ measurements of interstellar dust with the Ulysses and Galileo spaceprobes, Space Sci. Rev., 78, 165172, 1996.

Baranov, V. B., K. V. Krasnobaev, and M. S. Ruderman, On the model of the solar wind-local interstellar medium interaction with two shock waves, Astrophys. Space Sci., 41, 481-490, 1976.

Baranov, V. B. and Yu. G. Malama, Model of the solar wind interaction with the local interstellar medium: numerical solution of self-consistent problem, J. Geophys. Res., 98, 15157-15163, 1993.

Baranov, V. B. and Yu. G. Malama, Axisymmetric self-consistent model of the solar wind interaction with the LISM: basic results and possible ways of development, Space Sci. Rev., 78, 305-316, 1996.

Baroody, E. M., A theory of secondary electron emission from metals, Phys. Rev., 78, 780-787, 1950.

Bruining, H., Physics and Applications of Secondary Electron Emission, 128 pp., Pergamon Press, London, 1954.

Burke, E. A., Secondary emission from polymers, IEEE Trans. Nucl. Sci., NS-27, 1760-1764, 1980.

Caron, M., M. Beuve, H. Rothard, B. Gervais, A. Dubus, and M. Rösler, Experimental and theoretical study of target thickness dependent electron yields induced by electrons in carbon, Nucl. Instr. Meth. Phys. Res. B,
135, 436-442, 1998

Chow, V. W., The role of grain size in secondary and photoelectric emission from dust grains, in Advances in Dusty Plasmas, edited by P. K. Shukla, D. A. Mendis, and T. Desai, pp. 77-86, World Scientific, Singapore, 1997.

Chow, V. W., D. A. Mendis, and M. Rosenberg, Role of grain size and particle velocity distribution in secondary electron emission in space plasmas, $J$ Geophys. Res., 98, 19065-19076, 1993.

Chow, V. W., D. A. Mendis, and M. Rosenberg, Secondary emission from small dust grains at high electron energies, IEEE Trans. Plasma Sci., 22, 179-186, 1994.

Dorschner, J., Properties of interstellar dust, in Physics, Chemistry, and Dynamics of Interplanetary Dust, edited by B. A. S. Gustafson and M. S Hanner, pp. 487-496, Astronomical Society of the Pacific, San Francisco, 1996.

Draine, B. T., Photoelectric heating of interstellar gas, Astrophys. J. Suppl. Ser., 595-619, 1978

Draine, B. T., Charging processes for interstellar dust, in Evolution of Interstellar Dust and Related Topics, edited by A. Bonetti, J. M. Greenberg, and S. Aiello, pp. 91-101, Elsevier Science, New York, 1989.

Draine, B. T. and H. M. Lee, Optical properties of interstellar graphite and silicate grains, Astrophys. J., 285, 89-108, 1984.

Draine, B. T. and E. E. Salpeter, On the physics of dust grains in hot gas, Astrophys. J., 231, 77-94, 1979.

Fitting, H.-J., Transmission, energy distribution, and SE excitation of fast electrons in thin solid films, Phys. Stat. Sol. (a), 26, 525-535, 1974.

Frisch, P. C., Characteristics of nearby interstellar matter, Space Sci. Rev., 72, 499-592, 1995

Frisch, P. C., J. M. Dorschner, J. Geiss, J. M. Greenberg, E. Grün, M. Landgraf, P. Hoppe, A. P. Jones, W. Krätschmer, T. J. Linde, G. E. Morfill, W. Reach, J. D. Slavin, J. Svestka, A. N. Witt, and G. P. Zank, Dust in the local interstellar wind, Astrophys. J., 525, 492-516, 1999.

Gayley, K. G., G. P. Zank, H. L. Pauls, P. C. Frisch, and D. E. Welty, Oneversus two-shock heliosphere: constraining models with Goddard High Resolution Spectrograph Ly $\alpha$ spectra toward $\alpha$ Centauri, Astrophys. J., 487, 259-270, 1997.

Gelfort, St., H. Kerkow, R. Stolle, V. P. Petukhov, and E. A. Romanovskii, Secondary electron yield induced by slowly moving heavy ions, $\mathrm{Nucl}$. Instr. Meth. Phys. Res. B, 125, 49-52, 1997.

Gloeckler, G., L. A. Fisk, and J. Geiss, Anomalously small magnetic field in the local interstellar cloud, Nature, 386, 374-377, 1997.

Göller, J. R. and E. Grün, Calibration of the GALILEO/ULYSSES dust detectors with different projectile materials and at varying impact angles, Planet. Space Sci., 37, 1197-1206, 1989.

Greenberg, J. M., Interstellar dust, in Cosmic Dust, edited by J. A. M. McDonnell, pp. 187-294, Wiley-Interscience, New York, 1978.

Grün, E., B. Gustafson, I. Mann, M. Baguhl, G. E. Morfill, P. Staubach, A. Taylor, and H. A. Zook, Interstellar dust in the heliosphere, Astron. Astrophys., 286, 915-924, 1994.

Grün, E., M. Baguhl, D. P. Hamilton, J. Kissel, D. Linkert, G. Linkert, and R. Riemann, Reduction of Galileo and Ulysses dust data, Planet. Space Sci., 43, 941-951, 1995a.

Grün, E., M. Baguhl, N. Divine, H. Fechtig, D. P. Hamilton, M. S. Hanner, J. Kissel, B.-A. Lindblad, D. Linkert, G. Linkert, I. Mann, J. A. M. McDonnell, G. E. Morfill, C. Polanskey, R. Riemann, G. Schwehm, N. Siddique, P. Staubach, and H. A. Zook, Three years of Galileo dust data Planet. Space Sci., 43, 953-969, 1995 b.

Grün, E., M. Baguhl, N. Divine, H. Fechtig, D. P. Hamilton, M. S. Hanner, J. Kissel, B.-A. Lindblad, D. Linkert, G. Linkert, I. Mann, J. A. M McDonnell, G. E. Morfill, C. Polanskey, R. Riemann, G. Schwehm, N Siddique, P. Staubach, and H. A. Zook, Two years of Ulysses dust data, Planet. Space Sci., 43, 971-999, 1995c.

Grün, E., P. Staubach, M. Baguhl, D. P. Hamilton, H. A. Zook, S. Dermott, B. A. Gustafson, H. Fechtig, J. Kissel, D. Linkert, G. Linkert, R. Srama M. S. Hanner, C. Polanskey, M. Horanyi, B. A. Lindblad, I. Mann, J. A M. McDonnell, G. E. Morfill, and G. Schwehm, South-north and radial traverses through the interplanetary dust cloud, Icarus, 129, 270-288, 1997.

Hachenberg, O. and W. Brauer, Secondary electron emission from solids, Adv. Electron. Electron Phys., 11, 413-499, 1959

Hall, T. D. and W. W. Beeman, Secondary electron emission from beams of polystyrene latex spheres, J. Appl. Phys., 47, 5222-5225, 1976.

Hasselkamp, D., S. Hippler, A. Scharmann, and T. Schmehl, Electron emission from clean solid surfaces by fast ions, Ann. Phys., 47, 555-567, 1990 
Havnes, O., G. E. Morfill, and F. Melands $\emptyset$, Effects of electromagnetic and plasma drag forces on the orbit evolution of dust in planetary magnetospheres, Icarus, 98, 141-150, 1992.

Heroux, L., M. Cohen, and J. E. Higgins, Electron densities between 110 and $300 \mathrm{~km}$ derived from solar EUV fluxes of August 23, 1972, J. Geophys. Res., 79, 5237-5244, 1974

Higgins, J. E., The solar EUV flux between 230 and $1220 \AA$ on November 9, 1971, J. Geophys. Res., 81, 1301-1305, 1976.

Holzer, T. E., Interaction between the solar wind and the interstellar medium, Ann. Rev. Astron. Astrophys., 27, 199-234, 1989.

Horányi, M., S. Robertson, and B. Walch, Electrostatic charging properties of simulated lunar dust, Geophys. Res. Lett., 22, 2079-2082, 1995.

ICRU, ICRU Report 49, Stopping Powers and Ranges for Protons and Alpha Particles, 286 pp., International Commission on Radiation Units and Measurements, Bethesda, 1993

ICRU, ICRU Report 55, Secondary Electron Spectra from Charged Particle Interactions, 114 pp., International Commission on Radiation Units and Measurements, Bethesda, 1996.

Jacobsson, H., Fundamental processes in $\mathrm{SiO}_{2}$ under ion bombardment, Ph.D. dissertation, Chalmers University of Technology, 109 pp., Göteborg, 1993

Jacobsson, H. and G. Holmén, The dependence of $\mathrm{Si}$ and $\mathrm{SiO}_{2}$ electron emission on the angle of ion incidence, J. Appl. Phys., 74, 6397-6400, 1993.

Jacobsson, H. and G. Holmén, Electron emission from ion-bombarded $\mathrm{SiO}_{2}$ thin films, Phys. Rev. B, 49, 1789-1795, 1994.

Jones, A. P., W. W. Duley, and D. A. Williams, Interstellar extinction correlations, Mon. Not. Roy. Astron. Soc., 229, 213-221, 1987.

Jonker, J. L. H., On the theory of secondary electron emission, Philips Res. Rep., 7, 1-20, 1952.

Jurac, S., R. A. Baragiola, R. E. Johnson, and E. C. Sittler, Jr., Charging of ice grains by low-energy plasmas: Application to Saturn's $E$ ring, $J$. Geophys. Res., 100, 14821-14831, 1995.

Kanaya, K., S. Ono, and F. Ishigaki, Secondary electron emission from insulators, J. Phys. D.: Appl. Phys., 11, 2425-2437, 1978.

Katz, I., D. E. Parks, M. J. Mandell, J. M. Harvey, D. H. Brownell, Jr., S. S. Wang, and M. Rotenberg, A three dimensional dynamic study of electrostatic charging in materials, Contractor Report (NASA CR-135256), NASA Lewis Research Center, Cleveland, 1977.

Kimura, H. and I. Mann, The electric charging of interstellar dust in the solar system and consequences for its dynamics, Astrophys. J., 499, 454-462, $1998 \mathrm{a}$

Kimura, H. and I. Mann, Charging of dust in the very local interstellar medium, in Physics of Dusty Plasmas, edited by M. Horányi, S. Robertson, and B. Walch, pp. 321-328, American Institute of Physics, Woodbury, 1998b

Kimura, H. and I. Mann, Selection effects on interstellar dust in heliosphere, Adv. Space Res., 25(2), 299-302, 1999.

Kimura, H., I. Mann, and A. Wehry, Interstellar dust in the solar system, Astrophys. Space Sci., 264, 213-218, 1999.

Kollath, R., Sekundärelektronen-Emission fester Körper bei Bestrahlung mit Elektronen, in Electron-Emission . Gas Discharges I, edited by S. Flügge, pp. 232-303, Springer-Verlag, Berlin, 1956 (in German).

Krüger, H., E. Grün, D. P. Hamilton, M. Baguhl, S. Dermott, H. Fechtig, B. A. Gustafson, M. S. Hanner, M. Horányi, J. Kissel, B. A. Lindblad, D. Linkert, G. Linkert, I. Mann, J. A. M. McDonnell, G. E. Morfill, C. Polanskey, R. Riemann, G. Schwehm, R. Srama, and H. A. Zook, Three years of Galileo dust data II: 1993-1995, Planet. Space Sci., 47, 85-106, 1999a

Krüger, H., E. Grün, M. Landgraf, M. Baguhl, S. Dermott, H. Fechtig, B A. Gustafson, D. P. Hamilton, M. S. Hanner, M. Horányi, J. Kissel, B. A. Lindblad, D. Linkert, G. Linkert, I. Mann, J. A. M. McDonnell, G. E. Morfill, C. Polanskey, G. Schwehm, R. Srama, and H. A. Zook, Three years of Ulysses dust data: 1993-1995, Planet. Space Sci., 47, 363-383, $1999 b$

Laor, A. and B. T. Draine, Spectroscopic constraints on the properties of dust in active galactic nuclei, Astrophys. J., 402, 441-468, 1993.

Linde, T. J., T. I. Gombosi, P. H. Roe, K. G. Powell, and D. L. DeZeeuw, Heliosphere in the magnetized local interstellar medium: Results of a three-dimensional MHD simulation, J. Geophys. Res., 103, 1889-1904, 1998.

Lye, R. G. and A. J. Dekker, Theory of secondary emission, Phys. Rev., 107, 977-981, 1957.

Mann, I. and H. Kimura, Interstellar dust properties derived from mass density, mass distribution and flux rates in the heliosphere, J. Geophys.
Res., 2000 (in press).

Mathis, J. S. and G. Whiffen, Composite interstellar grains, Astrophys. J., 341, 808-822, 1989.

Meckbach, W., G. Braunstein, and N. Arista, Secondary-electron emission in the backward and forward directions from thin carbon foils traversed by $25-250 \mathrm{keV}$ proton beams, J. Phys. B.: Atom. Molec. Phys., 8, L344L349, 1975.

Meyer-Vernet, N., "Flip-flop" of electric potential of dust grains in space, Astron. Astrophys., 105, 98-106, 1982.

Mezger, P. G., J. S. Mathis, and N. Panagia, The origin of the diffuse galactic far infrared and sub-millimeter emission, Astron. Astrophys., 105, 372 388, 1982.

Millet, J. M. and J.-P. J. Lafon, Secondary-electron emission from porous solids, Phys. Rev. A, 52, 433-438, 1995.

Mukai, T., On the charge distribution of interplanetary grains, Astron Astrophys., 99, 1-6, 1981.

Mullan, D. J. and C. N. Arge, Structure of the heliospheric MHD bow shock Effects of ion-atom drifts, J. Geophys. Res., 101, 2535-2545, 1996.

Nerney, S., S. T. Suess, and E. J. Schmahl, Flow downstream of the heliospheric terminal shock: the magnetic field on the heliopause, J. Geophys. Res., 98, 15169-15176, 1993.

Pauls, H. L. and G. P. Zank, Interaction of a nonuniform solar wind with the local interstellar medium, J. Geophys. Res., 101, 17081-17092, 1996.

Pauls, H. L. and G. P. Zank, Interaction of a nonuniform solar wind with the local interstellar medium 2. A two-fluid model, J. Geophys. Res., 102, 19779-19787, 1997.

Ratkiewicz, R., A. Barnes, G. A. Molvik, J. R. Spreiter, S. S. Stahara, M. Vinokur, and S. Venkateswaran, Effect of varying strength and orientation of local interstellar magnetic field on configuration of exterior heliosphere: 3D MHD simulations, Astron. Astrophys., 335, 363-369, 1998

Reach, W. T. and F. Boulanger, Infrared emission from interstellar dust in the local interstellar medium, in The Local Bubble and Beyond, edited by D. Breitschwerdt, M. J. Freyberg, and J. Trümper, pp. 353-362, SpringerVerlag, Berlin, 1998.

Richardson, J. D., The heliosphere-interstellar medium interaction: One shock or two?, Geophys. Res. Lett, 24, 2889-2892, 1997.

Ritzau, S. M. and R. A. Baragiola, Electron emission from carbon foils induced by keV ions, Phys. Rev. B, 58, 2529-2538, 1998 .

Salow, H., Über den Sekundäremissionfaktor elektronenbestrahlter Isolatoren, Zeitschr. f. techn. Physik, 21, 8-15, 1940 (in German).

Santry, D. C. and R. D. Werner, Energy loss of ${ }^{4} \mathrm{He}$ ions in $\mathrm{Al}_{2} \mathrm{O}_{3}$ and $\mathrm{SiO}_{2}$, Nucl. Instr. Meth. Phys. Res. B, 14, 169-172, 1986.

Schou, J., Secondary electron emission from solids by electron and proton bombardment, Scanning Microscopy, 2, 607-632, 1988.

Sternglass, E. J., Theory of secondary electron emission under electron bombardment, Scientific Paper 6-94410-2-P9, Westinghouse Research Laboratories, Pennsylvania, 1957.

Suess, S. T., The heliopause, Rev. Geophys., 28, 97-115, 1990.

Svedhem, H., R. Münzenmayer, and H. Iglseder, Detection of possible interstellar particles by the HITEN spacecraft, in Physics, Chemistry, and Dynamics of Interplanetary Dust, edited by B. A. S. Gustafson and M. S Hanner, pp. 27-30, Astronomical Society of the Pacific, San Francisco, 1996.

Vernazza, J. E., E. H. Avrett, and R. Loeser, Structure of the solar chromosphere. II. the underlying photosphere and temperature-minimum region, Astrophys. J. Suppl. Ser., 30, 1-60, 1976.

Voreades, D., Secondary electron emission from thin carbon films, Surf. Sci., 60, 325-348, 1976

Washimi, H. and T. Tanaka, 3-D magnetic field and current system in the heliosphere, Space Sci. Rev., 78, 85-94, 1996.

Whang, Y. C., L. F. Burlaga, and N. F. Ness, Locations of the termination shock and the heliopause, J. Geophys. Res., 100, 17015-17023, 1995.

Whiddington, R., The transmission of cathode rays through matter, Proc. Roy. Soc. Lond. (A), 86, 360-370, 1912.

Yong, Y. C., J. T. L. Thong, and J. C. H. Phang, Determination of secondary electron yield from insulators due to a low-kev electron beam, J. Appl. Phys., 84, 4543-4548, 1998.

Young, J. R., Penetration of electrons in aluminum oxide films, Phys. Rev. 103, 292-293, 1956

Zank, G. P. and H. L. Pauls, Modelling the heliosphere, Space Sci. Rev., 78 95-106, 1996

Zank, G. P., H. L. Pauls, L. L. Williams, and D. T. Hall, Interaction of the solar wind with the local interstellar medium: A multifluid approach, $J$. Geophys. Res., 101, 21639-21655, 1996. 
Ziegler, J. F., Helium: Stopping Powers and Ranges in All Elemental Matter, 367 pp., Pergamon Press, New York, 1977.

Ziegler, J. F., Handbook of Stopping Cross-Sections for Energetic Ions in All Elements, 432 pp., Pergamon Press, New York, 1980.

Ziemann, P. J., P. Liu, D. B. Kittelson, and P. H. McMurry, Electron impact charging properties of size-selected, submicrometer organic particles, $J$
Chem. Phys., 99, 5126-5138, 1995.

H. Kimura (e-mail: kimura@linmpi.mpg.de) and I. Mann (e-mail: mann@gps.caltech.edu) 